\title{
Analysis of dispersion effect on a NRZ-OOK terrestrial free-space optical transmission system
}

\author{
Mohamed Bouhadda $^{1 *}$, Fouad Mohamed Abbou², Mustapha Serhani ${ }^{1}$, Fouad Chaatit $^{2}$ and Ali Boutoulout ${ }^{1}$
}

\begin{abstract}
Background: In this paper, the impact of the dispersion effect, due to atmospheric pressure and temperature, on NRZ-OOK terrestrial free-space optical transmission system is investigated. An expression for the dispersion parameter in FSO atmospheric channel is derived.

Results: The results show that the variation of the refractive index along the transmission path induces fluctuations of group velocity dispersion of the optical pulse resulting in broadening of the pulse duration. Simulation results show that at a propagation distance of $7.5 \mathrm{~km}$, the broadening ratio for input pulse duration of $300 \mathrm{fs}$ is approximately 2.39. Further, at a propagation distance of $7.5 \mathrm{~km}$, the remaining fraction of energy is approximately $40 \%$ for a $300 \mathrm{fs}$ input pulse duration. However, by increasing the transmitter input power, the effect of dispersion could be reduced. Namely, for a reference BER of $10^{-9}$, the maximum distance that it could be achieved is about 1 . $461 \mathrm{~km}$ for an input power of $1 \mathrm{~mW}$, while it is about $2.694 \mathrm{~km}$ for an input power of $4 \mathrm{~mW}$.

Conclusions: The results indicate that the effect of dispersion resulting from pressure and temperature increases with the propagation distance, which induces a high BER. However, the results show that it is possible to reach longer propagation distances with a lower BER by increasing the input power.
\end{abstract}

Keywords: Dispersion, Pulse broadening ratio, NRZ-OOK, BER, FSO

\section{Background}

Recently, free space optical communication technology has attracted much research because it has been successfully used in various applications such as satellite communication, deep-space probes and terrestrial communication. The free space optical communication offers remarkable advantages over the radio waves transmission, namely; high data transmission, unlicensed transmission, reduced interference and high security. Further, the capacity of FSO communication system has been successfully increased in recent years. In particular, an optical time division multiplexing system operating at $1.28 \mathrm{Tbit} / \mathrm{s}$ data transmission over a single-mode channel has been established [1]. According to [2], through free-space optical wireless systems, up to $2.5 \mathrm{Gbit} / \mathrm{s}$ of data, voice and video communications can be transmitted. FSO communication

\footnotetext{
* Correspondence: mohamed.bouhadda@usmba.ac.ma

${ }^{1}$ Moulay Ismail University, MACS Laboratory, Meknès, Morocco

Full list of author information is available at the end of the article
}

provides line of sight (LOS) communication thanks to its narrow transmit beamwidth and works in visible and IR spectrum. Furthermore, FSO communication systems are classified into terrestrial and space optical links which include building-to-building, ground-tosatellite, satellite-to-ground, satellite-to-satellite and satellite-to-airborne platforms (see $[3,4,5]$ ). Typical terrestrial communication wavelengths such as 808, 1064 or $1550 \mathrm{~nm}$ are applicable because they fall within the atmospheric transmission window in the absorption spectrum. As a result, the atmospheric loss due to absorption for these wavelengths turns out to be negligible as noted in $[6,7]$. However, and due the variation of the atmospheric pressure and temperature, the refractive index undergoes random fluctuations along the transmission path. This induces fluctuations of group velocity dispersion of the optical pulse, and results in either, broadening or compressing the pulse duration. The Pulse broadening limits the bit rate of optical link, and induces inter-symbol interference between adjacent 
pulses, which increases, bit error rate of the free space optical communication system.

In this paper, we propose an analytical expression for temporal pulse broadening, and we investigate the effects of atmospheric pressure and temperature on temporal broadening and study the effect of atmospheric dispersion on NRZ-OOK terrestrial free-space optical transmission system. The paper is organized as follows. In Theoretical analysis section, we present the Theoretical analysis needed for the study. In Results and discussions section, we discuss and analyze the obtained results. Conclusion section concludes the paper.

\section{Methods}

Dispersion phenomena can drastically affect the propagation of an optical beam by random fluctuations of the refractive index due to temperature and pressure variations along the optical propagation path. Based on the work presented in [8], the refractive index in the visible light and infrared domain can be described by the following expression

$$
n=1+77.6\left(1+7.52 \times 10^{-3} \lambda^{-2}\right) \frac{P_{h}}{T_{h}} \times 10^{-6}
$$

Here $\lambda$ is the optical wavelength in $\mu \mathrm{m}, P_{h}$ is the atmospheric pressure in Millibar, and $T_{h}$ is the temperature of the atmosphere in Kelvin. The gradient of standard atmospheric temperature as function of height can be expressed as [9];

$$
T_{h}= \begin{cases}288-6.5 h 10^{-3} & \text { for } 0 \mathrm{~km} \leq h<11 \mathrm{Km} \\ 216,5 & \text { for } 11 \mathrm{~km} \leq h<20 \mathrm{Km} \\ 216,5+(h-20000) 10^{-3} & \text { for } 20 \mathrm{~km} \leq h<32 \mathrm{Km}\end{cases}
$$

For the gradient of standard atmospheric pressure as function of height is given by [9];

$$
P_{h}=1013\left(\frac{(288-0.006 h)}{288}\right)^{5.255}
$$

Where $h$ is the altitude in meters. Further, when a group of optical waves with narrow range of wavelengths co-propagate along the optical propagation path, their resultant lightwave packet travels at the group velocity group $v_{g}$ defined by:

$$
v_{g}=\frac{\mathrm{c}}{\mathrm{n}-\lambda \frac{d n}{d \lambda}}
$$

Where $\mathrm{c}$ is the speed of light in vacuum. Using $L=$ $\mathrm{v}_{\mathrm{g}} \mathrm{T}_{\mathrm{FSO}}$, where $L$ is link length of the FSO medium, we can obtain an expression of the pulse delay $\tau_{\mathrm{FSO}}$ as

$$
\tau_{F S O}=\frac{L}{C}\left(1+77.6\left(1+22.56 \times 10^{-3} \lambda^{-2}\right) \frac{P_{h}}{T_{h}} \times 10^{-4}\right)
$$

Further, considering an optical laser source with rms spectral width $\Delta \lambda$, the rms pulse broadening due to FSO medium can be derived as:

$$
\sigma_{F S O}=\Delta \lambda\left|\frac{d \tau_{F S O}}{d \lambda}\right|=3501.31 \Delta \lambda \frac{L}{c} \lambda^{-3} \frac{P_{h}}{T_{h}} 10^{-7}
$$

Hence, the FSO medium dispersion coefficient can be expressed as:

$$
D_{F S O}=-3501,31 \frac{\lambda^{-3}}{c} \frac{P_{h}}{T_{h}} 10^{-7}
$$

Hence, the third order $\beta_{3}$ and the second order $\beta_{2}$ derivatives of propagation constant $\beta$ can be expressed as:

$$
\begin{aligned}
& \beta_{2}=\frac{\lambda^{-1}}{2 \pi c^{2}} 3501,31 \frac{P_{h}}{T_{h}} 10^{-7} \\
& \beta_{3}=\frac{10503,93}{4 \pi^{2} c^{3}} \frac{P_{h}}{T_{h}} 10^{-7}
\end{aligned}
$$

$\beta_{2}$ is the group velocity dispersion (GVD), is known to be the primary source of pulse broadening [10]. The frequency dependence of the group velocity results in pulse broadening because different spectral components of the pulse disperse during propagation due to frequency chirps generated by the GVD induced phase shift.

Further, by ignoring the channel losses induced by scattering and absorption in a terrestrial FSO link, the received signal power $P_{r}$ at a distance $L$ with a transmitter signal power $P_{t}$ can be written as

Table 1 System parameters

\begin{tabular}{ll}
\hline Parameters & Value \\
\hline Transmission Wavelength $(\lambda)$ & $1550 \mathrm{~nm}$ \\
Distance $(\mathrm{L})$ & $1-10 \mathrm{~km}$ \\
Transmitter power $\left(P_{t}\right)$ & $1-50 \mathrm{~mW}$ \\
Optical Efficiency of Transmitter $\mathrm{T}_{\mathrm{t}}$ & 0.75 \\
Optical Efficiency of Receiver $\mathrm{T}_{\mathrm{r}}$ & 0.75 \\
Full transmitting divergence angle $\theta$ & $2^{*} 10^{-3} \mathrm{rad}$ \\
Receiver Diameter & $1 \mathrm{~cm}$ \\
Electron Charge $(\mathrm{q})$ & $1.6 \times 10^{-19} \mathrm{C}$ \\
PIN Load Resistance $(\mathrm{R})$ & $1 \mathrm{k} \Omega$ \\
Boltzmann Constant $(\mathrm{k})$ & $1.38 \times 10^{-23} \mathrm{~J} . \mathrm{k}$ \\
Temperature $(T)$ & $298 \mathrm{~K}$ \\
Dark Current $\left(\mathrm{I}_{\mathrm{d}}\right)$ & $10 \mathrm{nA}$ \\
Responsivity $\left(\mathrm{R}_{\mathrm{d}}\right)$ & $0.6 \mathrm{~A} / \mathrm{W}$ \\
Bandwidth $(B)$ & $0.5 \mathrm{GHz}$ \\
\hline
\end{tabular}




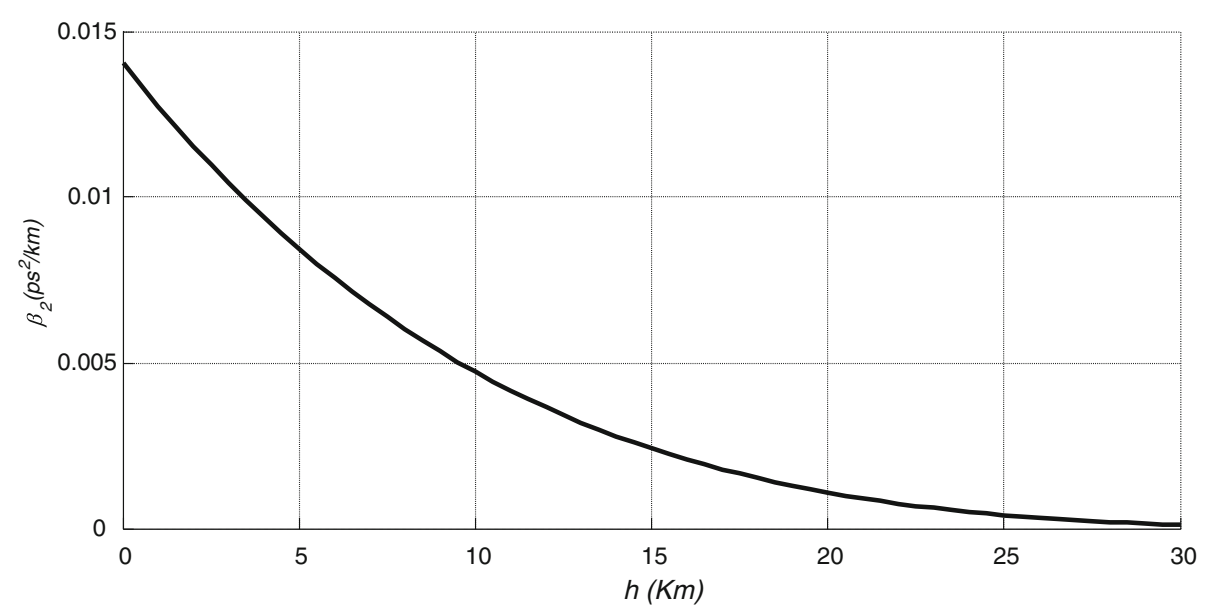

Fig. 1 The second order dispersion coefficient $\beta_{2}$ as a function of the altitude

$$
P_{r}=P_{t}\left(\frac{D}{L \theta}\right)^{2} \tau_{t} \tau_{r}
$$

Where $D$ is the receiver diameter, $\theta$ is the full transmitting divergence angle, and $\tau_{r}$ and $\tau_{t}$ are the optical efficiencies of the transmitter and the receiver respectively. In order to evaluate the FSO performance in the presence of dispersion, the SNR and BER are considered. For a PIN photodiode receiver, the signal-to-noise ratio (SNR) can be written as

$$
\frac{S}{N}=\frac{\left(P_{r} R_{d}\right)^{2}}{i_{d}^{2}+i_{t h}^{2}+i_{s h}^{2}}
$$

Where $i_{d}^{2}$ is the detector dark noise, $i_{t h}^{2}$ is the thermal noise and $i_{s h}^{2}$ is the shot noise. The noise sources are expressed mathematically by:

$$
\begin{aligned}
& i_{d}^{2}=2 q B I_{d} \\
& i_{s h}{ }^{2}=2 q B I_{p} \\
& i_{t h}{ }^{2}=\frac{4 k T B}{R}
\end{aligned}
$$

Where $I_{p}=P_{r} R_{d}$ is the average photocurrent, $R_{d}$ is the receiver responsivity, $q$ is the charge of an electron, $B$ represents the bandwidth, $T$ is the absolute photodiode temperature (K), and $R$ is the PIN load resistor, and $\mathrm{k}$ is the Boltzmann's constant. The NRZ-OOK Bit Error Rate (BER) of a FSO link can be expressed as

$$
\mathrm{BER}=\frac{1}{2} \operatorname{erfc}\left(\frac{1}{2 \sqrt{2}} \sqrt{\frac{S}{N}}\right)
$$

In the next section, simulation results will be discussed to analyze the effect of dispersion on FSO optical

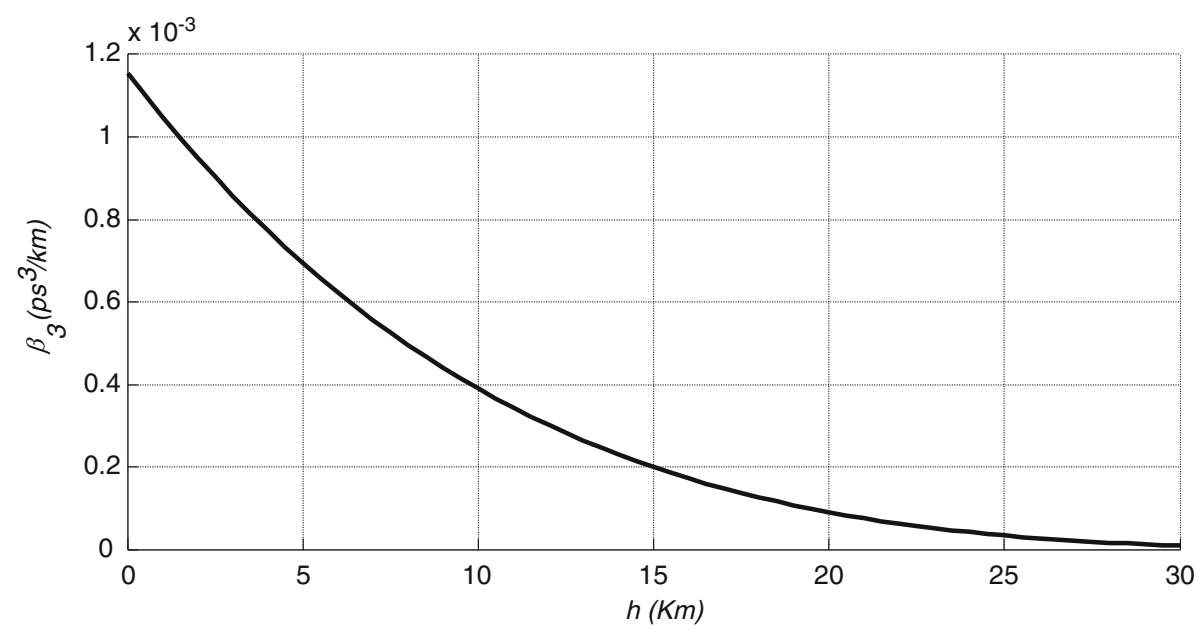

Fig. 2 The third order dispersion coefficient $\beta_{3}$ as a function of the altitude 


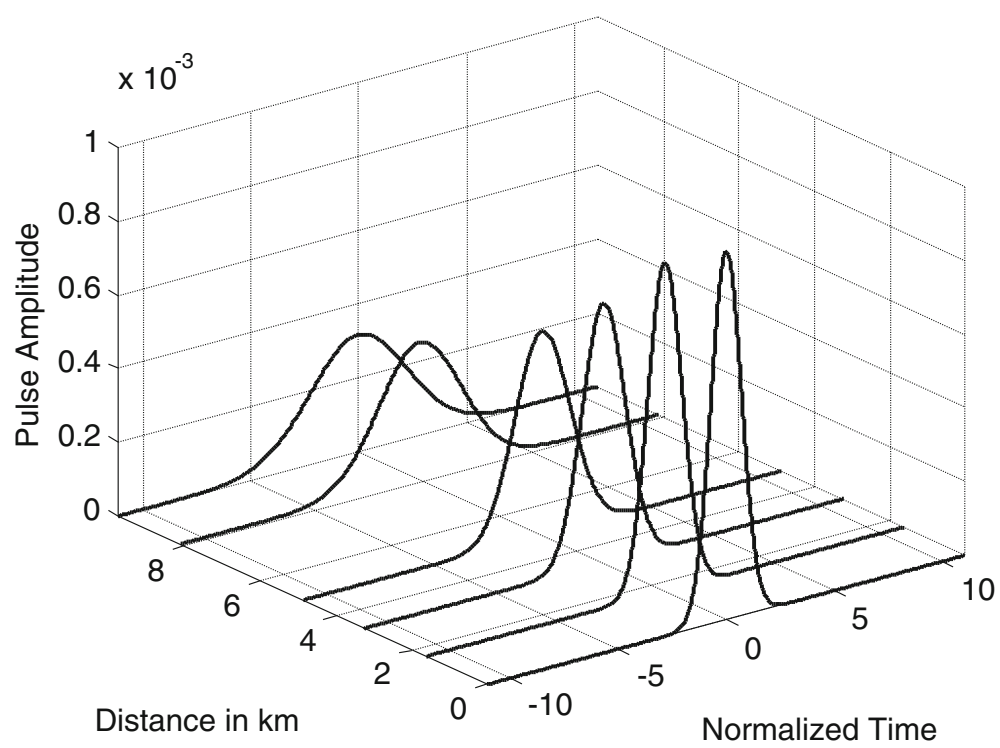

Fig. 3 Pulse propagation at $z=10 \mathrm{~km}$ and with $\beta_{2}=0.002 \mathrm{ps}^{2} / \mathrm{km}$

wireless communication system employing NRZ-OOK modulation technique. The simulation parameters are defined in Table 1.

\section{Results and discussions}

Following the theoretical analysis presented early on, the effect of dispersion due to atmospheric pressure and temperature on a terrestrial free-space optical communication system is investigated. Figures 1 and 2 show the curves of second and third order dispersion coefficients as a function of the altitude. Clearly, it can be seen that both $\beta_{2}$ and $\beta_{3}$ are decreasing with the altitude. From Fig. 3 , it is obvious that the GVD induced pulse broadening increases linearly with the propagation distance and therefore imposes limitation on the FSO link. Further, the pulse broadening ratio as a function of propagation distance, for different input pulses is depicted in Fig. 4. It is clear from Fig. 4 that the broadening ratio increases with the propagation distance. At a propagation distance of $7 \mathrm{~km}$, the values of the broadening ratios for the three different input pulses with $\mathrm{T}_{0}=300 \mathrm{fs}, \mathrm{T}_{0}=400 \mathrm{fs}$, and $\mathrm{T}_{0}=500 \mathrm{fs}$, are

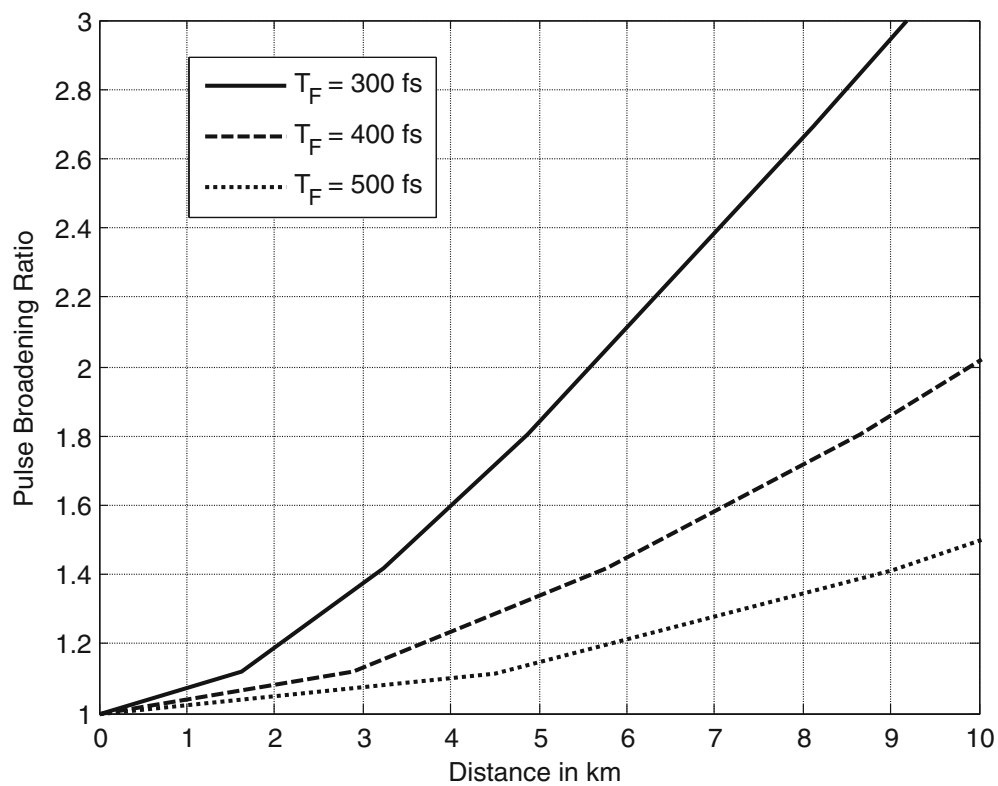

Fig. 4 Broadening ratio as a function of propagation distance for different input pulses 


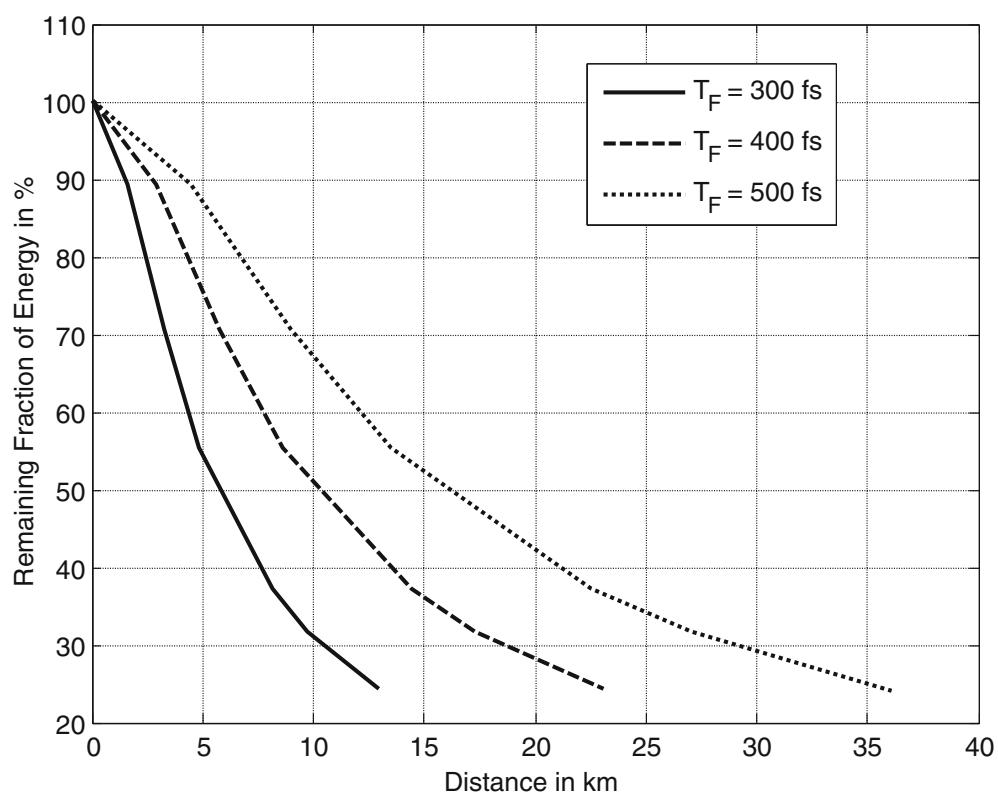

Fig. 5 Remaining fraction of energy as a function of the propagation distance

found to be approximately $2.39,1.59$ and 1.29 respectively. This is obvious as short pulsed are most sensitive to dispersion effect.

Further as shown in Fig. 5, the pulse remaining fraction of energy decreases with the distance due to the attenuation induced by dispersion. For example, at a propagation distance of $7.5 \mathrm{~km}$, the remaining fraction of energy is approximately $40 \%$ for a $300 \mathrm{fs}$ input pulse. Thus, it is quite obvious that at a large link distance, it is difficult to maintain sufficient pulse energy. However, from Fig. 6, the BER curves for NRZ-OOK modulation format for different values of input power show that by increasing the transmitter input power, the effect of dispersion could be reduced and therefore it would be possible to achieve longer propagation distance with significant lower BER.

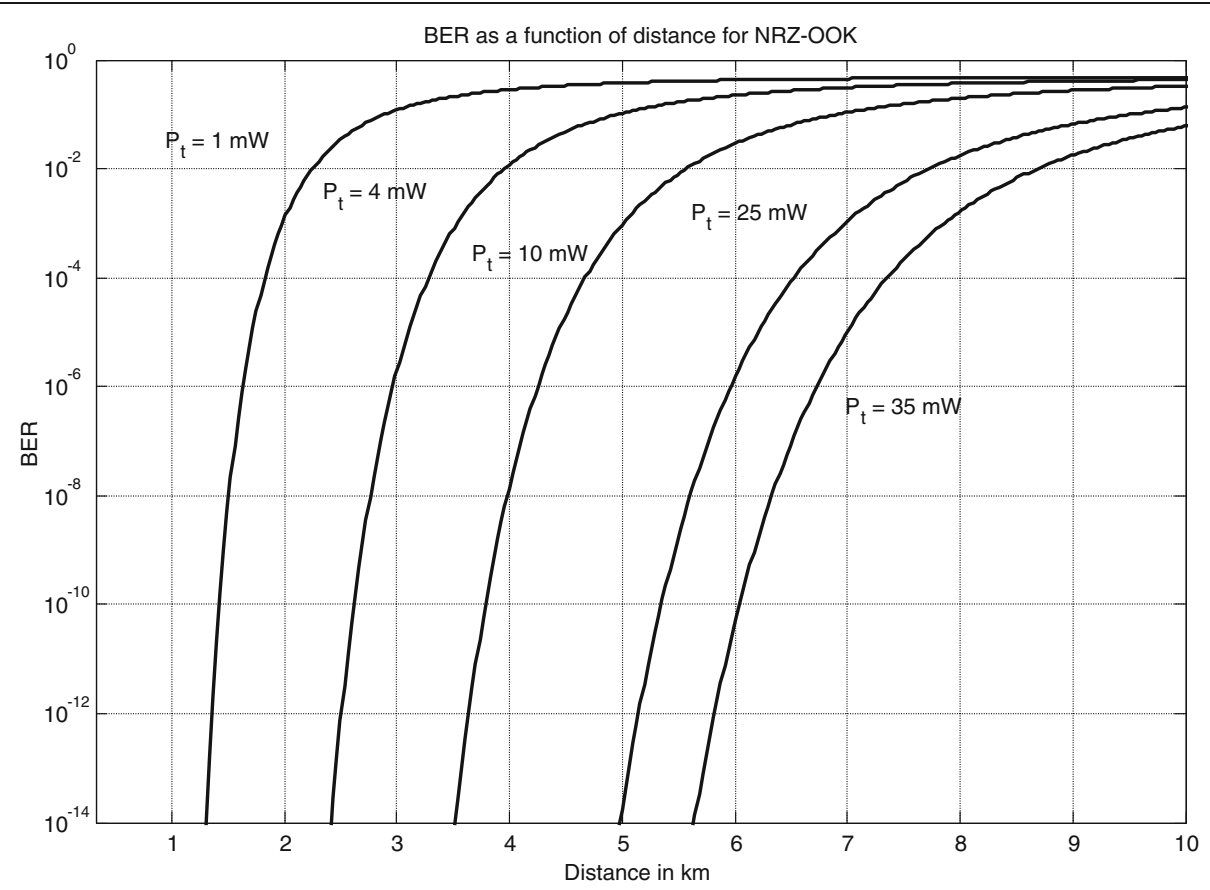

Fig. 6 NRZ-OOK BER versus link distance for different values of transmitter power 


\section{Conclusion}

The effect of dispersion due to atmospheric pressure and temperature on a terrestrial free-space optical communication system is semi-analytically analyzed. A general expression for the medium dispersion coefficient due to pressure and temperature is derived. It is clear that the dispersion effect due to pressure and temperature increases with the propagation distance. At a propagation distance of $7.5 \mathrm{~km}$, the remaining fraction of energy is approximately $40 \%$ for a 300 fs input pulse. Further, performance results show that the dispersion induced pulse broadening limits the link distance and induces high BER. However, by increasing the transmitter input power, the effect of dispersion could be reduced and therefore it would be possible to achieve longer propagation distance with significant lower BER.

\section{Acknowledgement}

The authors would like to extend their special thanks and appreciations to the Al Akhawayen University and Moulay Ismail University, Morroco for supporting this work.

\section{Authors' contributions}

$\mathrm{MB}$ and FMA participated in the development of the mathematical model andcarried out the simulation. MB and FMA, MS, FC and $\mathrm{AB}$ contributed in the analysis of the results. All authors helped to draft the manuscript. All authors have read and approved the final manuscript.

\section{Competing interests}

The authors declare that they have no competing interests.

\section{Author details}

${ }^{1}$ Moulay Ismail University, MACS Laboratory, Meknès, Morocco. ${ }^{2} \mathrm{Al}$

Akhawayen University, School of Sciences and Enginnering, Ifrane, Morocco.

Received: 26 May 2016 Accepted: 7 October 2016

Published online: 17 October 2016

\section{References}

1. Howlader, MK, Jung, J: Inter-symbol interference due to the atmospheric turbulence for free-space optical communication system. In: IEEE International Conference on Communications, p. 5046. (2007)

2. FSONA Systems Corp: Unveils 2-5-Gbps free-space optical systems. (2012)

3. Ghassemlooy, Z, Popoola, WO: Terrestial Free-Space Optical Communications, ch. 17. In: Fares, SA, Adachi, F (eds.). pp. 356-392. European Union, Rijeka (2010). ISBN 978-953-307-042-1

4. Sharma, V, Kumar, N: Improved analysis of 2.5 Gbps-inter-satellite link (ISL) in inter-satellite optical wireless communication (ISOWC) system. Opt. Commun. 286, 99-102 (2014)

5. Majumdar, AK, Ricklin, JC: Free-Space Laser Communications: Principles and Advanced, springer science+business media. LLC, New York (2008)

6. Henninger, H, Wilfert, O: An Introduction to Free-space Optical Communications. Radio Eng. 19(2), 203-212 (2010)

7. Alkholidi, A, Altowii, K: Effect of Clear Atmospheric Turbulence on Quality of Free Space Optical Communications in Western Asia, Das, N. (ed.) Optical Communications Systems, ISBN: 978-953-51-0170-3, InTech (2012). doi:10.5772/35186

8. Andrews, LC, Phillips, RL: Laser beam propagation through random media, 2nd edn. SPIE Optical Engineering Press, Bellingham (2005)

9. Manual of the ICAO Standard Atmosphere Doc 7488/3, International Civil Aviation Organization, 3rd edn. (1993)

10. Govind, P: Agrawal Fiber-Optic Communication Systems, 2nd edn. (1997)

\section{Submit your manuscript to a SpringerOpen ${ }^{\odot}$ journal and benefit from:}

- Convenient online submission

Rigorous peer review

- Immediate publication on acceptance

- Open access: articles freely available online

- High visibility within the field

Retaining the copyright to your article 